\title{
Induration Longest Diameter
}

National Cancer Institute

\section{Source}

National Cancer Institute. Induration Longest Diameter. NCI Thesaurus. Code C122196.

The longest diameter of an area of hardness in the skin. 